Mappemonde

Revue trimestrielle sur l'image géographique et les formes du territoire

$120 \mid 2017$

Varia

\title{
Le jardin tropical de Paris
}

\section{Clarisse Didelon-Loiseau}

URL : https://journals.openedition.org/mappemonde/3056

DOI : 10.4000/mappemonde.3056

ISSN : 1769-7298

\section{Éditeur}

UMR ESPACE

\section{Référence électronique}

Clarisse Didelon-Loiseau, «Le jardin tropical de Paris », Mappemonde [En ligne], 120 | 2017, mis en

ligne le 01 avril 2017, consulté le 28 juin 2022. URL : http://journals.openedition.org/mappemonde/ 3056 ; DOI : https://doi.org/10.4000/mappemonde.3056

Ce document a été généré automatiquement le 29 septembre 2020

\section{(c) (i) (2)(2)}

La revue Mappemonde est mise à disposition selon les termes de la Licence Creative Commons Attribution - Pas d'Utilisation Commerciale - Partage dans les Mêmes Conditions 4.0 International. 


\title{
Le jardin tropical de Paris
}

\author{
Clarisse Didelon-Loiseau
}

1 Aux portes de Paris, enfin de l'autre côté du bois de Vincennes, il est un jardin oublié. Un dimanche après-midi d'été, alors qu'au même moment le lac Daumesnil connait un embouteillage au ponton de location des barques, on n'y croise guère plus d'une demi-douzaine de personnes. Désorientés, les visiteurs marchent avec précaution, presque sur la pointe des pieds, et parlent bas pour ne pas troubler la quiétude de ce jardin à la végétation folle. Nous avons pénétré dans un jardin parisien à l'apparence extérieure relativement classique avec sa grille marron-vert et ses horaires d'ouverture clairement affichés. Mais, sitôt passée la grille, nous nous sommes trouvés face à une porte chinoise ${ }^{1}$ sous laquelle chemine une allée ombragée de grands arbres. Puis, au détour d'un sentier, nous sommes tombés sur un ensemble de débris de statues (photographie 1). L'une d'elles sans tête, la poitrine dénuée ornée d'un collier de plusieurs rangs de perles arbore une pose qui fut lascive. Un Asiatique, vidant une grande jarre, lève sa tête vers le ciel ou vers un personnage qui fut là. Sur un reste de bas-relief, un coq se dresse fièrement sur un globe sous lequel gisent des outils (ou des armes ?). Une figure féminine (la République ?) coiffée de laurier, l'air grave, semble étendre sa cape pour protéger quelque chose; mais on ne distingue rien sous la cape.

Photographie 1. Ensemble de statues

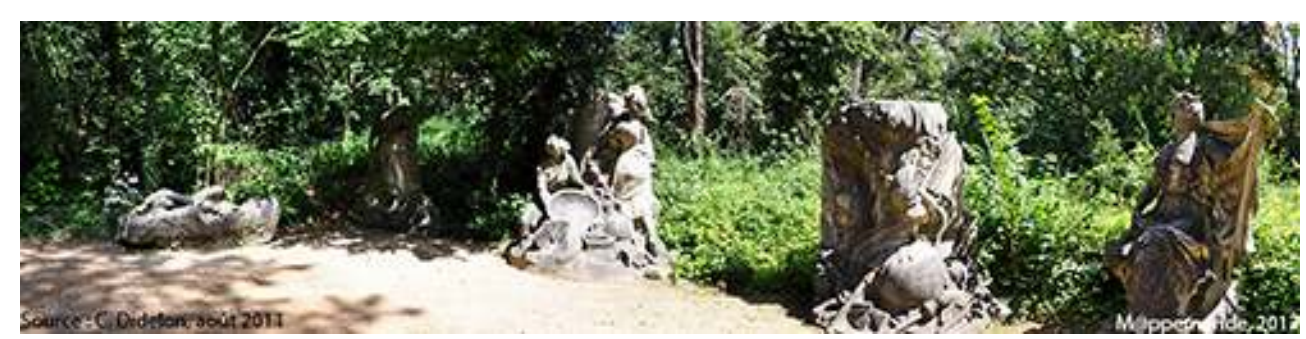

Source : Clarisse Didelon, 2011

2 Puis, dans une petite clairière bordée d'une anarchie de jeunes noisetiers, à quelques minutes à pied de la station de RER « Nogent-sur-Marne », se dresse comme un stupa de «style indochinois ${ }^{2}$. Et dans la chaleur de cet après-midi d'été, il nous faut regarder 
les arbres à deux fois, pour nous assurer que nous sommes bien à Paris et que nous n'avons pas été transportés sans nous en rendre compte dans la jungle d'une contrée lointaine. Mais, déjà, voici d'autres surprises. Après avoir traversé un petit pont khmer, nous voici sur une vaste place où, face aux ruines d'un portique vietnamien, se dresse une pagode rouge vif, apparemment récente, au milieu d'une pelouse bien entretenue. Entre les deux, une grande urne funéraire. Nous sommes sur l'esplanade du Dinh (photographie 2); nous sommes au jardin tropical de Paris.

Photographie 2. Esplanade du Dinh

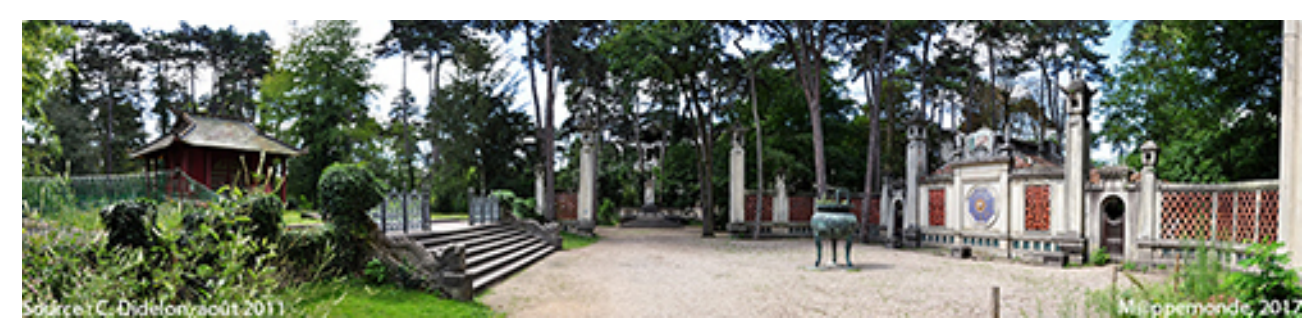

Source : Clarisse Didelon, août 2011

Contrairement à ce que son nom indique, il ne faut pas s'attendre à trouver ici trace d'une quelconque végétation tropicale. Seules subsistent, çà et là, quelques bambouseraies à proximité des pièces d'eau. De la végétation tropicale, seul le nom conserve la mémoire. Mais ce lieu a bel et bien été, et c'était sa raison d'être, un jardin d'essai colonial. Ouvert en 1899 , les semences des plantes tropicales y étaient étudiées et améliorées avant d'être renvoyées dans les colonies. Ce fut, par exemple, de ce jardin qu'ont été initiées les plantations de riz au Sénégal. Le jardin se dote bientôt d'une école (l'École nationale supérieure d'agronomie coloniale, en 1902) et sa vocation de formation des cadres coloniaux et de la recherche en agronomie tropicale va s'affirmer peu à peu. Jusqu'après le démantèlement des colonies. Caféiers, cacaoyers, hévéas, vanilliers, muscadiers, bananiers, plants de cotons et de quinquina étaient chez eux au bois de Vincennes. Aujourd'hui les serres du jardin d'essai tropical sont rouillées (photographie 3) et les jeunes branches de vigoureuses plantes endémiques s'en échappent par les vitres brisées. 


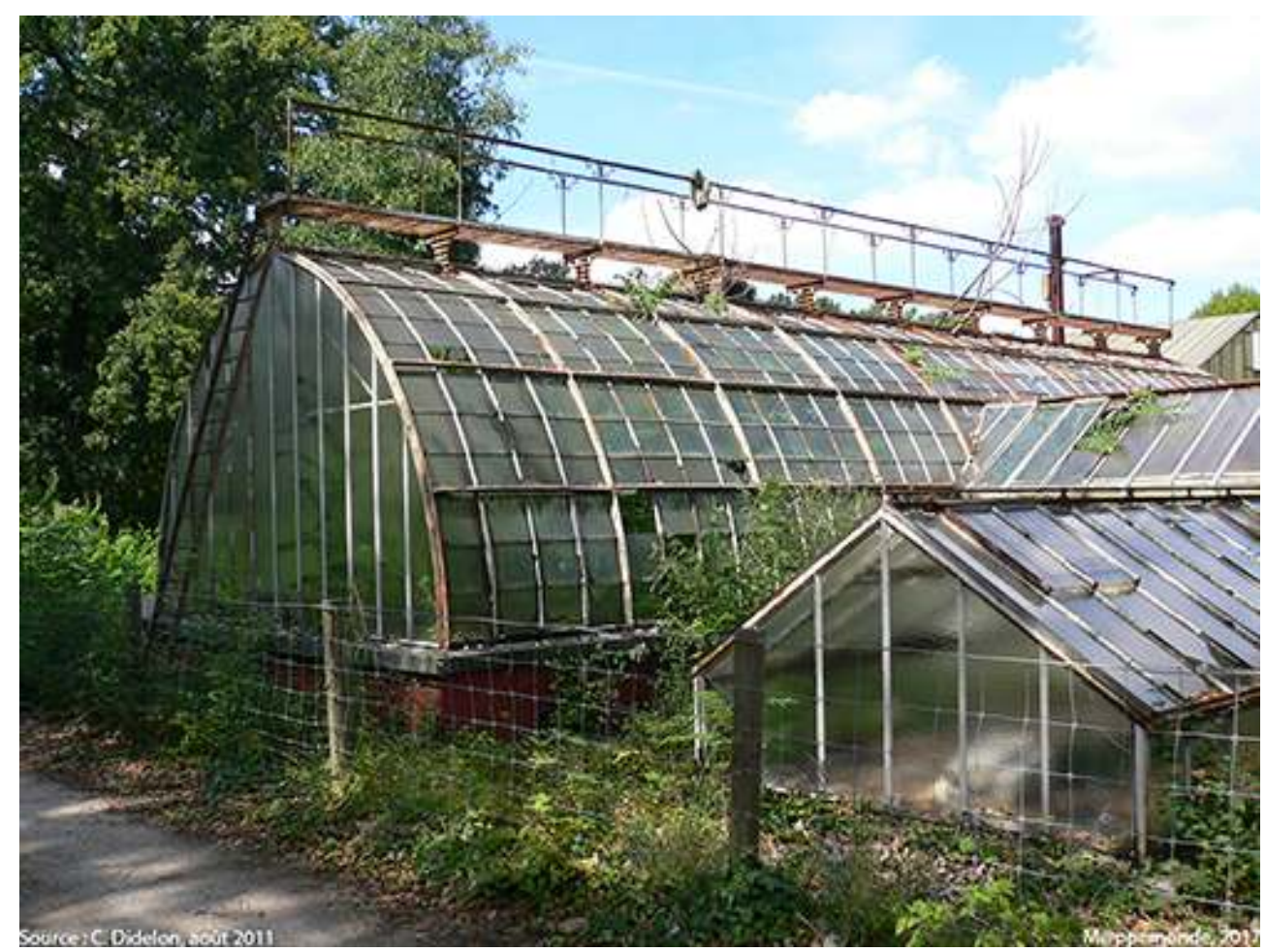

Source : Clarisse Didelon, août 2011

En 1907, le jardin accueille pour quelque mois une exposition coloniale, consacrée exclusivement aux colonies françaises (http://www.expositions-universelles.fr). De cette époque datent la plupart des pavillons exotiques qui jalonnent les allées. Mais on $\mathrm{y}$ trouvait également des reconstitutions de villages indigènes, cinq en tout (indochinois, malgache, kanak, béninois, congolais) avec leurs monuments, leurs productions, leurs habitats et. leurs habitants. Il y aurait eu aussi une ferme du Soudan, un campement touareg (avec attaque du courrier) et même un centre de dressage d'éléphants. En six mois, deux millions de visiteurs se sont pressés devant ces reconstitutions "de carton-pâte" auxquelles les indigènes, salariés et recrutés sur contrat, étaient chargés de donner vie. Nous sommes alors dans une autre époque, celle où les scientifiques européens, en particulier ceux issus de l'anthropologie physique, construisaient des théories sur la "hiérarchie des races ", que l'on retrouvait jusque dans les livres d'école (figure $\mathbf{1}^{3}$ ). Cette théorisation scientifique de la suprématie de la race blanche intervient alors que le rôle de l'Église décroit et que les Lumières ont mis à mal son discours sur la mission de christianisation des populations indigènes par l'homme blanc (Bessis, 2001). L'empire colonial est alors en pleine construction et les expositions coloniales mettent en œuvre le « mécanisme colonial de l'infériorisation de l'indigène » dans ce que l'on appelle désormais des « zoos humains » tandis que, dans le même temps, elles assouvissent la passion d'exotisme de la population. Comme le résument Bancel et al. (2004) : "Premiers phénomènes de masse du $19^{\mathrm{e}}$ siècle avec les expositions universelles et leurs millions de visiteurs, les zoos humains donnent une réalité au discours racial alors en construction $»^{4}$. Ainsi, le jardin tropical est l'une des illustrations du mécanisme de la construction de «l'autre » colonial décrit par Edward Said (2005). Pourtant, il ne faut pas perdre de vue que, au fur et à mesure que les attractions indigènes ont gagné en notoriété, elles ont été de plus en plus décriées, 
notamment par la presse liée au parti colonial qui «n'apprécie pas cet exotisme de pacotille qui nuit au sérieux des vastes ambitions impériales de la III République " (Pierre et Hodeir, 2011). En tout état de cause, ces expositions ont été pour leur contemporains les "plus vivante[s] des leçons de choses " (Lyautey, cité par Pierre et Hodeir, 2011), et elles constituaient un outil d'information relativement efficace à destination des populations, à une époque où les images du reste du monde étaient beaucoup moins accessibles qu'aujourd'hui.

Figure 1. «Les quatre races d'hommes »

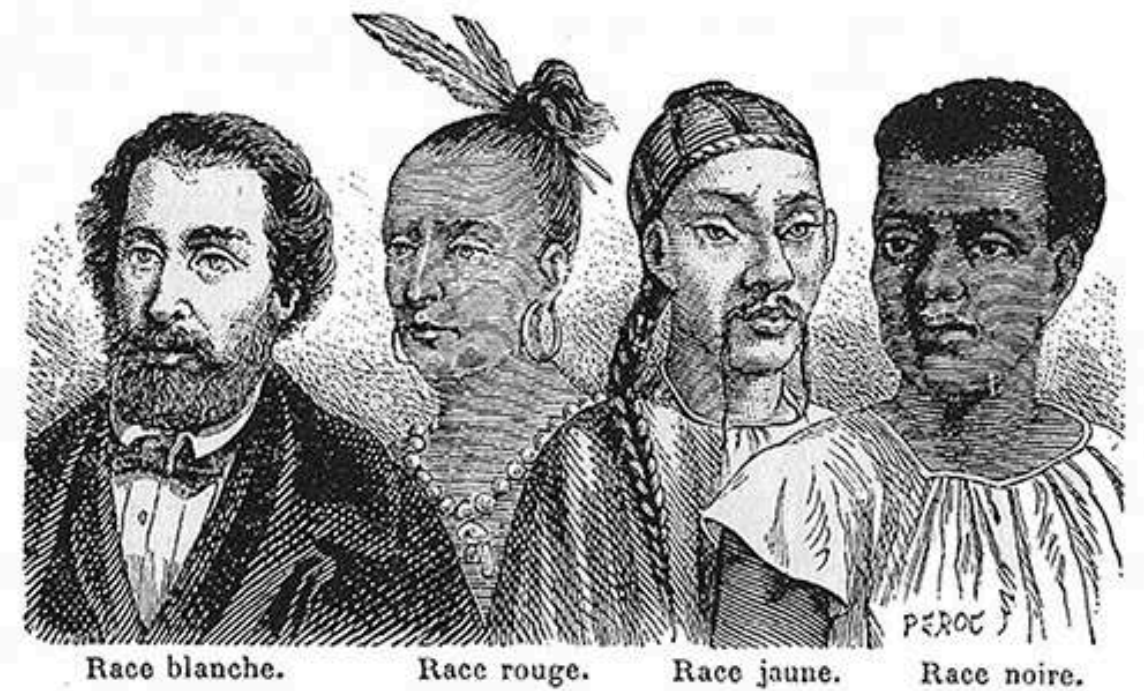

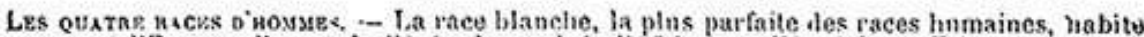

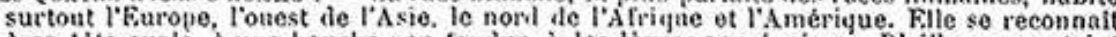
à sa téte ovale, a une bouche pen fondue, à les lèvres pen épaissos. D'aillenrs son teint peut varier. - La mee jaune occupe prinkipalement J'Asicorientale, la Chine et le Japon : visage plat, pommettes saillantes, nez aptati. paupierres briulees, yenx en amandes, pou de chevenx et peu de barbe. - Ja race rouge, qui habitait autrefois tonte I rifue, a une peau rouzedtre, les youx enfoncés, le nez long et aryné, lo front très fuyant. - La race noire, yui ocenje startont lifrique et lo sul de l'Océanie, a la peau très noire, les clieveux crépus, le noz ètasé, les lèvres êpaisses, les bras très longs.

Source: G. Bruno - Le tour de France par deux enfants - 1976

Meppemonde, 2017

Source : G. Bruno - Le tour de France par deux enfants - 1976

Les quatre races d'hommes - La race blanche, la plus parfaite des races humaines, habite surtout l'Europe, l'Ouest de l'Asie, le nord de l'Afrique et l'Amérique. Elle se reconnaît à sa tête ovale, à une bouche peu fendue, à des lèvres peu épaisses. D'ailleurs son teint peut varier. - La race jaune occupe principalement l'Asie orientale, la Chine et le Japon : visage plat, pommettes saillantes, nez aplati, paupières bridées, yeux en amandes, peu de cheveux et peu de barbe. - La race rouge, qui habitait autrefois toute l'Amérique, a une peau rougeâtre, les yeux enfoncés, le nez long et arqué, le front très fuyant. - La race noire, qui occupe surtout l'Afrique et le sud de l'Océanie, a la peau très noire, les cheveux crépus, le nez écrasé, les lèvres épaisses, les bras très longs.

De cette exposition coloniale, la mémoire s'efface peu à peu. Si elle dû être splendide, ce qu'il reste des bâtiments qu'elle abritait illustre la difficulté de faire face à ce pan de l'histoire de France. Les pavillons d'exposition sont aujourd'hui dans un état variable illustrant pratiquement l'état des relations entre la France et ses anciennes colonies. Certains sont flambants neufs, restaurés comme la maison d'Indochine 
(photographie 4). D'autres, comme le pavillon du Maroc (photographie 5), insalubres et dangereux, sont entourés de barrières et de rubans de travaux pour en interdire l'accès. D'autres ont été brûlés récemment dans des incendies criminels comme celui du Congo, dont il ne reste guère que quelques poutres sinistres s'élevant vers le ciel (photographie 6). Cela a également été le cas de la pagode de l'esplanade du Dinh, brûlée en 1984 après un pillage en règle et remplacée par la pagode actuelle, plus modeste $^{5}$. Le pavillon de la Tunisie (photographie 7), agrandi et percé de fenêtres, a quant à lui servi de laboratoire de physique quand le site accueillait encore les chercheurs. Aujourd'hui, il est à nouveau désaffecté et ses encadrements de fenêtres accueillaient en août 2011 d'étranges sculptures dont on pouvait se demander s'il s'agissait d'art ou simplement d'étais dessinant un motif complexe. La serre du Dahomey est couverte d'une bâche bleue; le pavillon de la Réunion d'une bâche transparente. Tous deux doivent faire face aux assauts de la végétation locale.

Photographie 4. Pavillon d'Indochine

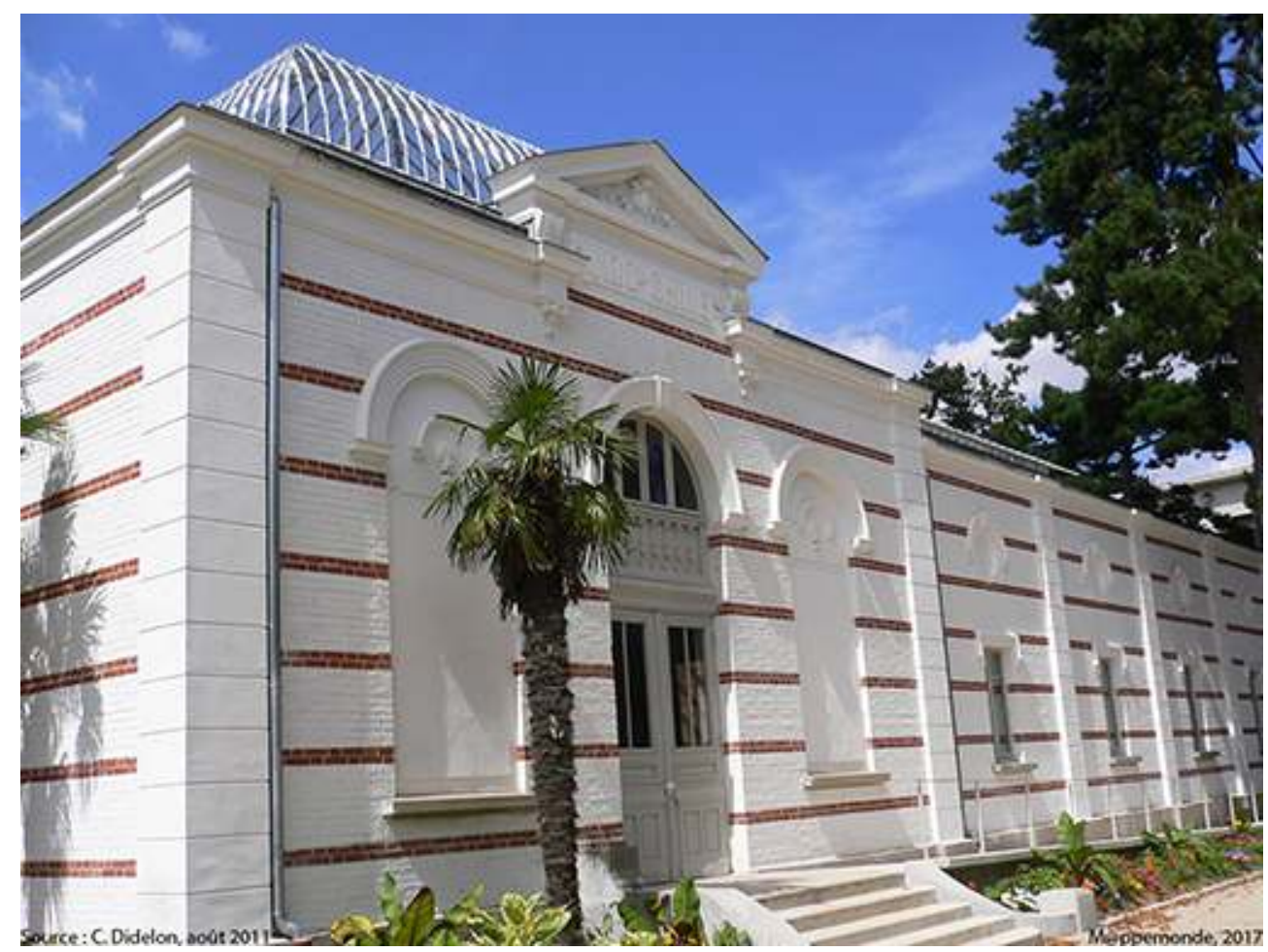

Source : Clarisse Didelon, août 2011 
Photographie 5. Pavillon du Maroc

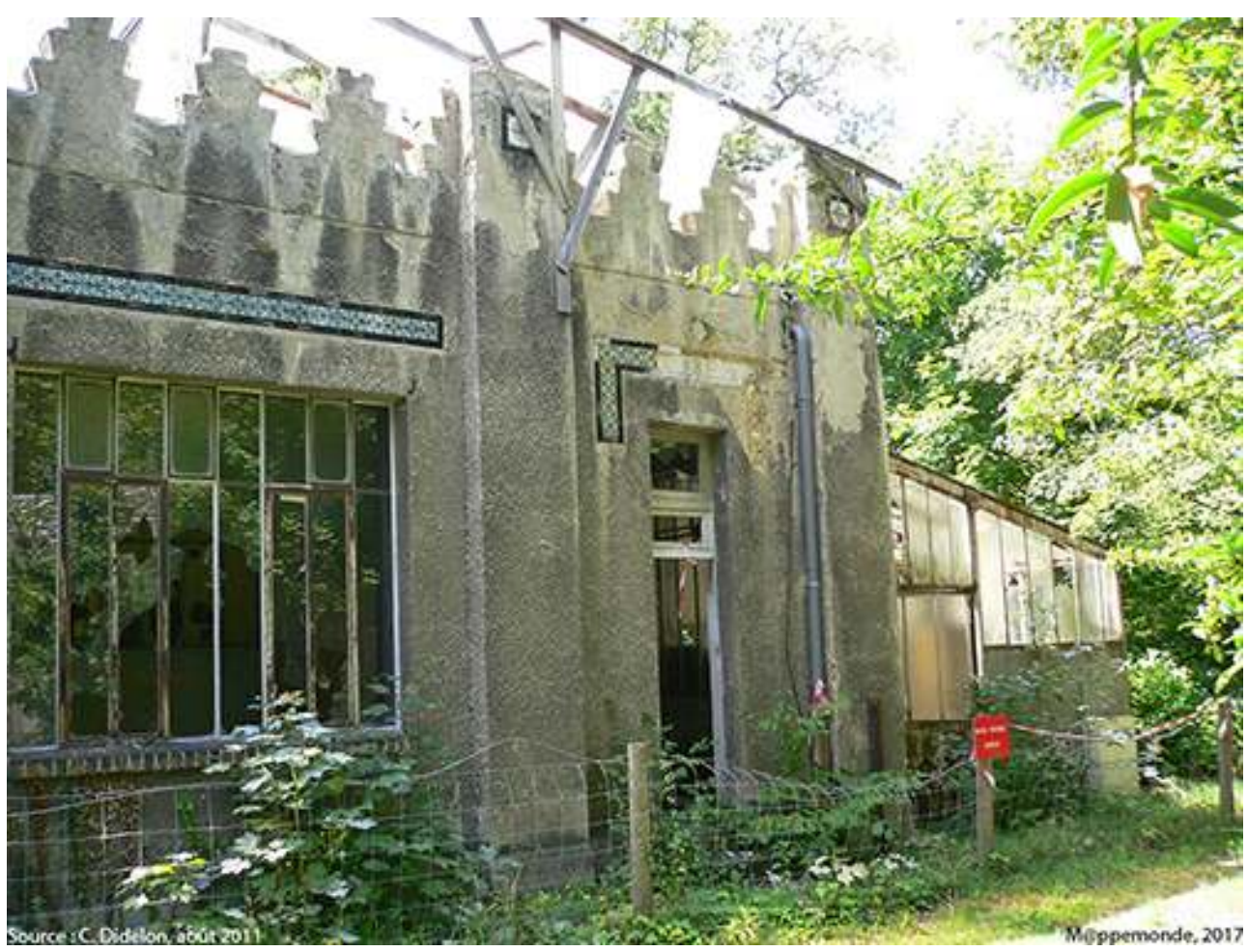

Source : Clarisse Didelon, août 2011

Photographie 6. Pavillon du Congo

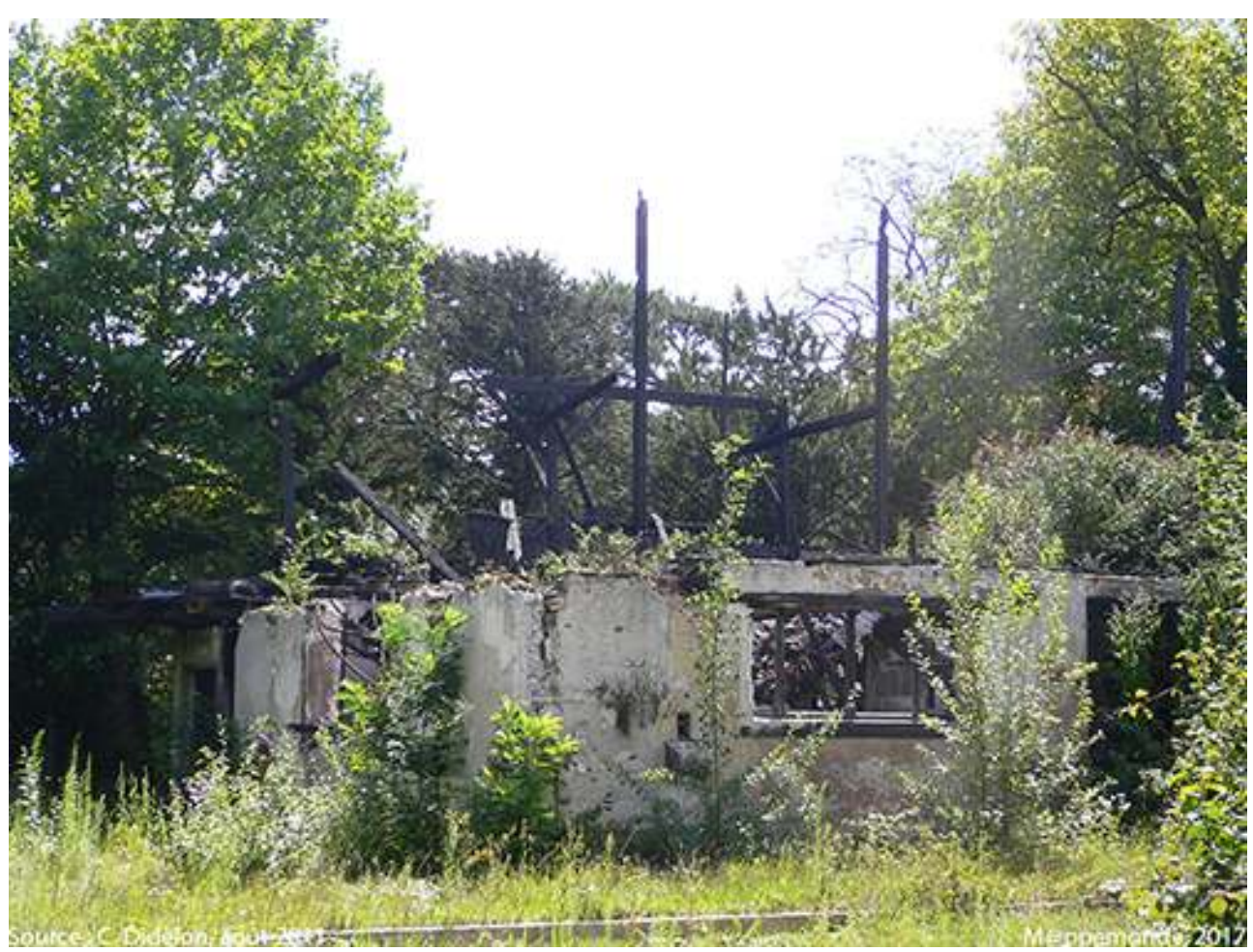

Source : Clarisse Didelon, août 2011 
Photographie 7. Pavillon de la Tunisie

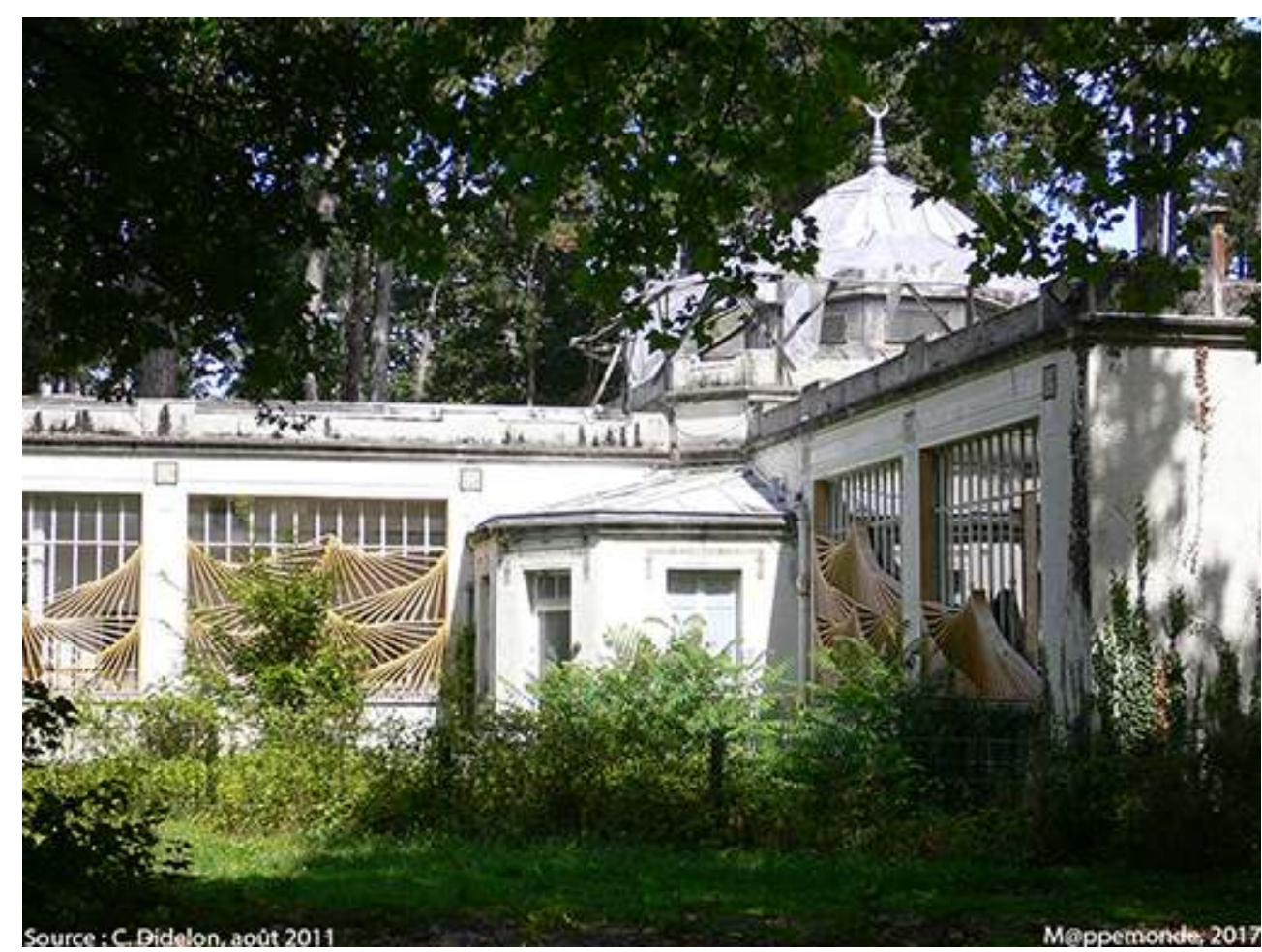

Source : Clarisse Didelon, août 2011

Quant à la mosquée, ne la cherchez pas. Si la première mosquée de Paris a bien été construite ici en 1916, il n'en reste rien qu'une plaque commémorative à l'endroit où elle s'élevait. Mais pourquoi une mosquée à l'autre bout du bois de Vincennes? Parce que pendant la Première Guerre mondiale le site a servi d'hôpital pour accueillir les soldats blessés ou malades des troupes coloniales. Cette mise à l'écart étonne aujourd'hui. Craignait-on alors que les soldats coloniaux soient porteurs de quelque maladie? Voulait-on leur apporter un peu de réconfort en leur permettant de se retrouver entre eux ? De cette guerre d'ailleurs, moult souvenirs pathétiques jalonnent les allées : les monuments aux morts se dressant à la mémoire des soldats coloniaux. Certains sont dédiés à quelques catégories particulières des populations coloniales comme les Indochinois chrétiens (photographie 8). Mais la plupart forment un inventaire des «morts pour la France » qui fait le tour du monde : aux «Cambodgiens et Laotiens "; « à la mémoire des Vietnamiens » (dans toutes les guerres françaises de 1924 à 1962 !) ; « aux soldats noirs » (photographie 9); «au souvenir des soldats de Madagascar». Un monument, enfin, s'élève au milieu d'une prairie d'herbes folles à la mémoire des "soldats coloniaux de la Grande Guerre" provenant de toutes les contrées de la France d'Outre-mer, soigneusement listées et classées par continents, autre catégorie mentale inventée par les Européens (Grataloup, 2009): France d'Afrique, France d'Amérique, France d'Asie et France d'Océanie. Le plan du jardin reflète d'ailleurs dans cette catégorisation du monde en aires géographiques et lieux de civilisations. Ainsi, le nord du jardin concentre les monuments asiatiques tandis que le sud regroupe les monuments africains et guyanais. À l'intérieur même de ces zones des séparations sont effectuées, par exemple entre le monde bouddhique indianisé et le monde vietnamien sinisé confucéen. 
Photographie 8. Monument aux morts « aux Indochinois chrétiens »

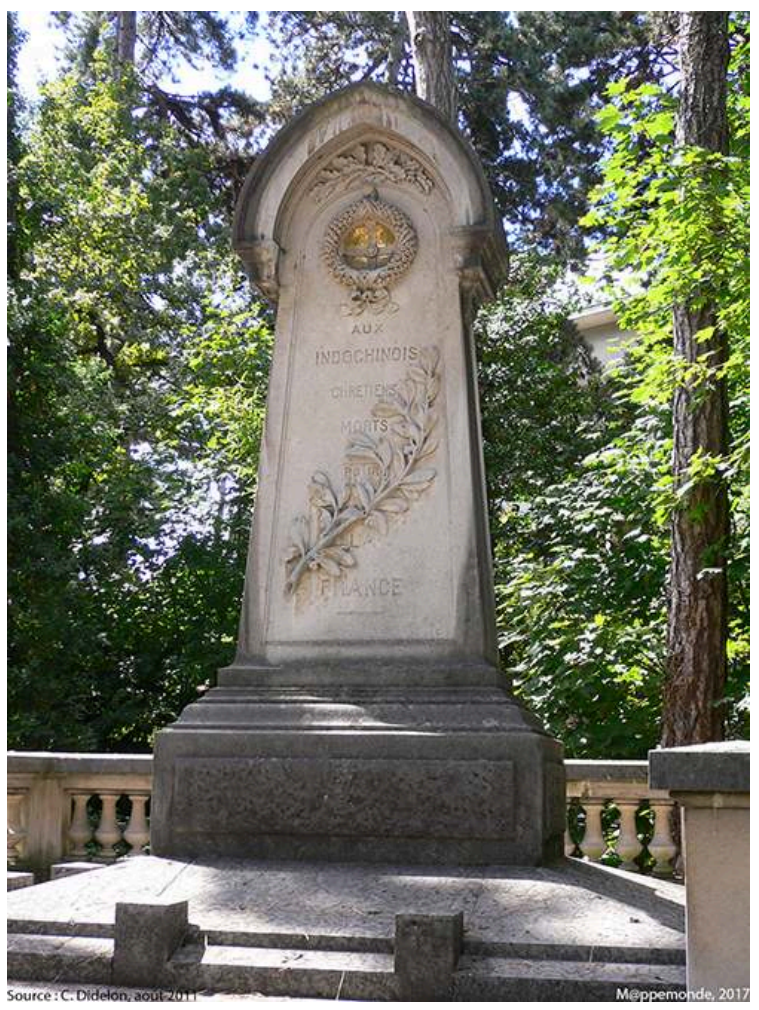

Source : Clarisse Didelon, août 2011

Photographie 9. Monument aux morts « aux soldats noirs »

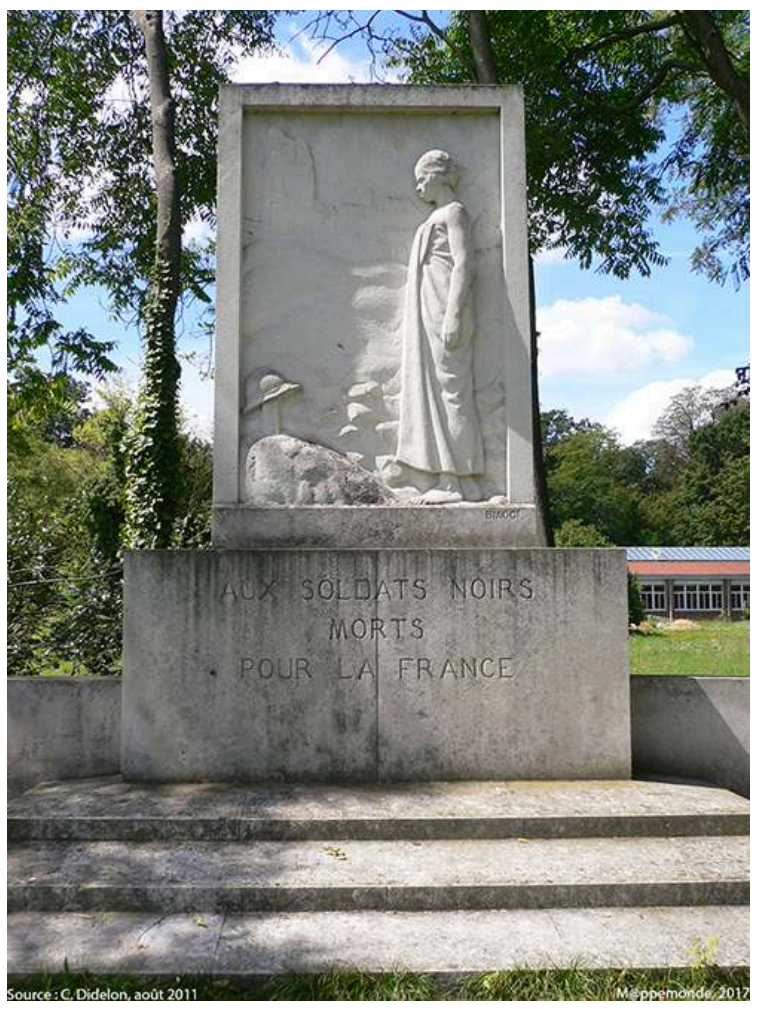

Source : Clarisse Didelon, août 2011 
Mais ce jardin est décidément plein de surprises : à l'ombre des arbres on trouve aussi deux monuments à la mémoire de personnalités françaises. La première, Eugène Étienne (1844-1921) fut un inspecteur des chemins de fer et sénateur de la troisième République, un leader du "parti colonial », un groupe de pression puissant de la III République, à visée impérialiste et "dont le principal moteur intellectuel était constitué par le mouvement géographique animé par les membres des sociétés de géographie " (http://etudescoloniales.canalblog.com). Plusieurs fois sous-secrétaire d'État aux colonies, Eugène Etienne œuvra au développement de l'empire français, en invoquant la recherche du profit pour justifier l'expansion coloniale (Andurain, 2008). Non loin de sa statue financée par la ligue maritime et coloniale française, une plaque à la mémoire de René Dumont, (1904-2001), qui fût élève de l'Institut national d'agronomie coloniale puis enseignant à l'École supérieure d'agronomie tropicale. Ces deux personnages sont contemporains respectivement du début et de la fin de l'empire colonial républicain. La statuaire reflète ainsi les deux facettes de la posture occidentale face à la colonisation : «tantôt l'Occident se rengorge de sa supériorité et en use pour asservir les vaincus, tantôt il cède à une autocritique culpabilisante (...) qui nourrira les «tiers-mondismes dévots des années 60-70» (Guillebaud, 2008). À côté de ces plaques et statues, perdue dans ce bric-à-brac de la mémoire coloniale, la statue d'un jeune apollon se couvre doucement de lierre.

Le jardin tropical fut donc un espace dédié au développement des colonies françaises et il a abrité certains des épisodes les moins glorieux de l'histoire coloniale se jouant en métropole, comme les zoos humains. L'exposition coloniale de 1907 a parfaitement incarné les deux penchants d'une pensée binaire, "mépriser l'autre ou le célébrer » (Guillebaud, 2008), oscillant entre la mise en scène de représentants de catégories raciales jugées inférieures et "l'admiration éblouie à l'endroit des coutumes, des traditions et des arts primitifs », (id.) qui caractérise le "goût des autres » (de l'Estoile, 2007). Avec ses monuments qui s'effondrent peu à peu et sa végétation folle, le jardin tropical prend aujourd'hui l'allure d'une friche malgré son classement en 1994 à l'Inventaire des monuments historiques. D'une vraie friche, mais également d'une friche mémorielle (Pelletier, 2010), puisqu'il incarne la trace problématique d'un passé dont on ne peut se glorifier mais pour lequel on ne souhaite pas avoir honte. Entre nostalgie de la gloire passée et honte d'un passé peu glorieux, le jardin tropical fait presque figure de secret de famille, commodément situé à distance à l'autre bout du bois de Vincennes. Et, non loin de l'ancien palais des colonies de l'Exposition coloniale de 1931 (un autre lieu mémoriel problématique aujourd'hui transformé en musée de l'immigration), ce cimetière des colonies françaises fait le bonheur des photographes amateurs en mal d'exotisme.

Jardin tropical de Paris, 45 avenue de la Belle Gabrielle, 75012 Paris. 


\section{BIBLIOGRAPHIE}

ANDURAIN (d') J. (2008). « Réseaux d'affaires et réseaux politiques : le cas d'Eugène Étienne et d'Auguste d'Arenberg ». In BONIN H., KLEIN J., HODEIR C. (dir), L'esprit économique impérial, groupes de pression et réseaux du patronat colonial en France et dans l'Empire, Revue française d'histoire d'outremer, SFHOM, p. 85-102.

BANCEL et al. (2004). Zoos humains. Au temps des exhibitions humaines. Paris : La découverte/Poche, 490 p. ISBN 9782707144010

BANCEl N., BLANCHARD P., Lemaire S. (2000). « Ces zoos humains de la république coloniale ». Le Monde diplomatique. Consulté en février 2017.

BESSIS S. (2001). «L'Occident et les autres. Histoire d'une suprématie ». Paris : La découverte/ Poche, 350 p. ISBN 9782707142559

HERBELIN C. (2010). Architecture et urbanisme en situation coloniale : le cas du Vietnam. Thèse de doctorat en Histoire de l'art, Université Paris 4.

L'ESTOILE B. de (2007). Le goût des autres. De l'Exposition coloniale aux arts premiers. Paris :

Flammarion, 454 p. ISBN 9782082104982

GRATALOUP C. (2009). L'invention des continents. Paris, Larousse, coll. « Terre \& Nature », 224 p. ISBN $978-2035825940$

GUILLEBAUD J.-C. (2008). Le commencement d'un monde. Paris : Seuil, 400 p. ISBN 2020967073

PELLETIER B. (2010). Le jardin tropical de Paris, une friche mémorielle de la France coloniale. En ligne. Consulté en février 2017.

PIERRE M., HODEIR C. (1999). L'Exposition coloniale. Bruxelles : Éditions Complexe, coll. « La mémoire du siècle », 159 p. ISBN 2870273827

SAID E.W. (2005). L'Orientalisme. L'Orient créé par l'Occident. Paris : Seuil, coll. « La couleur des idées », 430 p. EAN 9782020792936

\section{Sites Internet}

http://www.expositions-universelles.fr

http://etudescoloniales.canalblog.com

http://www.baudelet.net/paris/jardin-tropical.htm

http://www.anai-asso.org

\section{NOTES}

1. En travaux lors de notre visite.

2. En réalité, comme le souligne notre relecteur, «il n'existe pas de "stupa de style indochinois" "; le "style indochinois » n'ayant existé que dans l'esprit des colonisateurs et de quelques architectes du service des travaux publics de l'Indochine (cf. thèse de Caroline Herbelin)». 
3. La planche du livre proposée ici n'est pas particulièrement originale et les productions de ce type sont assez courantes dans la littérature enfantine et les manuels scolaires. Toutefois ici, le commentaire ne laisse aucune place à l'ambigüité sur la supériorité de la " race blanche ». Par ailleurs, la date de réédition de livre (1976) laisse songeur.

4. La thèse des "zoos humains » est une interprétation fortement remise en cause par la communauté historienne. Toutefois, l'exposition du Musée du quai Branly («Exhibitions, l'invention du sauvage » de décembre 2011 à août 2012) et la publicité qui en a été faite dans le journal du CNRS ( $n^{\circ} 263$, déc. 2011) ont montré sa vivacité et sa diffusion auprès du grand public. 5. « C'était un très bel édifice d'architecture traditionnelle vietnamienne, construit sur le modèle d'un « dinh », maison communale, offert par la province de Thudaumot pour servir de maison de la Cochinchine à l'exposition coloniale de Marseille en 1906, puis à l'exposition coloniale de Nogent-sur-Marne en 1907.

Racheté par le gouvernement français, il avait été mis en 1917 à la disposition du Souvenir Indochinois ». Source :

http://www.anai-asso.org/NET/document/lieux_de_memoire/

les_temples_du_souvenir_indochinois/index.htm

INDEX

Mots-clés : exposition coloniale, histoire coloniale, jardin, lieu de mémoire, lieux

\section{AUTEUR}

CLARISSE DIDELON-LOISEAU

Université Paris 1 